\title{
Predictive Ability of the Stability and Workload Index for Transfer Score to Predict Unplanned Readmissions After ICU Discharge*
}

\author{
Marc Kastrup, $\mathrm{MD}^{1}$; Robert Powollik, $\mathrm{MD}^{1}$; Felix Balzer, $\mathrm{MD}^{1}$; Susanne Röber, $\mathrm{MD}^{1}$; Robert Ahlborn ${ }^{1}$; \\ Vera von Dossow-Hanfstingl, MD, $\mathrm{PhD}^{2}$; Klaus D. Wernecke, MD, PhD ${ }^{3}$; Claudia D. Spies, MD, $\mathrm{PhD}^{1}$
}

\begin{abstract}
Objective: Unplanned readmission of hospitalized patients to an ICU is associated with an increased mortality and hospital length of stay. The ability to identify patients at risk, who would benefit from prolonged ICU treatment, is limited. The aim of this study is to validate a previously published numerical index named the Stability and Workload Index for Transfer in a heterogeneous group of ICU patients.

Design: In this retrospective data analysis, the Stability and Workload Index for Transfer score was calculated for all patients, and the ability of the score to predict readmission was compared with the original publication.
\end{abstract}

Setting: Four ICUs, one intermediate care unit, and one postanesthesia care unit of the department of anesthesia and intensive care of a university hospital.

Patients: All consecutive patients treated in one of the units.

Interventions: None.

Measurements and Main Results: Unplanned ICU readmissions or unexpected death within 7 days of ICU discharge. The data of 7,175 patients were included in the analysis. Five hundred ninety-six patients were readmitted or died within 7 days of discharge. The patients who are readmitted to the ICU are significantly older and have significantly higher scores that define the severity of disease at the time of admission and discharge of their first ICU stay. The

\footnotetext{
*See also p. 1803.

${ }^{1}$ Charité - Universitätsmedizin Berlin, Charitéplatz 1, Berlin, Germany.

${ }^{2}$ Klinikum Großhadern, Ludwig Maximilians Universität München, München, Germany.

${ }^{3}$ Sostana $\mathrm{GmbH}$, Berlin, Germany.

Dr. Spies provides expert testimony: Ethical Committee Vienna Faculty of Medicine, grants pending: Abbott, Aspect, Baxter, Carefusion, Deltex, Fresenius, Grunenthal, Hutchison, Kohler Chermie, MSD, MCN, Novartic, Pajunk, Pulsion, Roche, Sysmex, University Hospital Stavenger, Argus, BDA, BMBF, DKH, DLR, German Reseach Society, GIZ, Inner University grants, Stifterverband, payment for lectures: Abbott, Essex Pharma, GSK, payment for manuscript prep: Bispebjerg Hospital, travel: Abbott, Aspect. The remaining authors have disclosed that they do not have any potential conflicts of interest.
}

For information regarding this article, E-mail: claudia.spies@charite.de Copyright (C) 2013 by the Society of Critical Care Medicine and Lippincott Williams \& Wilkins

DOI: 10.1097/CCM.0b013e31828a217b source of admission for the initial ICU stay did not differ $(p=0.055)$, and the last Glasgow Coma Scale and the last $\mathrm{PaO}_{2} / \mathrm{FIO}_{2}$ ratio before discharge from the ICU were higher in patients who did not need a readmission to the ICU. The performance of the Stability and Workload Index for Transfer score is poor with an area under the receiver operator curve of $0.581(95 \% \mathrm{Cl}, 0.556-0.605 ; p<0.001)$.

Conclusions: Based on the data from our patients, the proposed Stability and Workload Index for Transfer score by Gajic et al is not ideal in aiding the clinician in the decision, if a patient can be discharged safely from the ICU and further research is necessary to define the patients at risk for readmission. (Crit Care Med 2013; 41:1608-1615)

Key Words: discharge; intensive care unit; prediction score; readmission; risk

S ome critically ill patients experience unexpected clinical deterioration, which results in a readmission or death shortly after discharge from the ICU. Large studies on ICU readmission report readmissions between $2.0 \%$ and $6.4 \%$ $(1-7)$. Besides the differences of the definition within the studies, lower readmission rates may depend on several factors, including the permanent presence of an intensivist responsible for discharge decisions, variations in patient population, ratio of ICU beds to hospital beds, and the availability of stepdown units. Early but appropriate discharge from the ICU reduces excessive and unnecessary use of this expensive healthcare facility and allows the admission of patients in need for intensive monitoring and care (1). Discharge decisions are influenced not only by the status of the patient but also by workload pressure and bed demand (8). Early discharge of ICU patients to general wards increases the risk for ICU readmission $(9,10)$. Patients who are readmitted to the ICU have an increased mortality rate of 1.5-10 times compared with controls and at least twice the length of hospital stay of control patients $(1,6,9,11)$.

Some early readmissions are avoidable. The proportion of preventable readmissions varies in the different studies within 
a range from $5 \%$ to $50 \%$. Whatever the exact figure is, the readmission rates include a significant fraction of events that could have been avoided $(12,13)$. The knowledge of risk factors for ICU readmission might help identify high-risk patients, who will profit from intensive care treatment $(6,9,14)$. A large-scale retrospective cohort study with ICU data from American ICUs demonstrated that ICU readmission is associated with certain patient factors that reflect a greater severity and complexity of illness and results in a higher risk for hospital mortality and a longer hospital stay (6). Several tools have been proposed before determining whether discharge is appropriate. A rating scale, based on a subjective assessment of the treating physician, has been reported to predict hospital mortality after ICU discharge (15). In several countries, critical care outreach teams are used to detect patients who need to be readmitted $(16,17)$. Tools or scores based on objective data could help decide whether a patient can be discharged safely or a special surveillance after ICU treatment is justified. The Stability and Workload Index for Transfer (SWIFT) score was developed by Gajic et al (18) in 2008 to predict unplanned readmission. In two validation cohorts, a North American medical ICU and a European surgical-medical ICU, the score was used to predict readmission. This score was derived from information readily available at the time of ICU discharge. Chandra et al (19) used an automated system based on the electronic medical record to calculate the risk of unplanned readmission using the SWIFT score for medical ICU patients, and they were able to demonstrate the reliability and excellent correlation with manual data collection.

The aim of this study is to demonstrate whether the calculation of the SWIFT score can predict unplanned readmission and death within 7 days after discharge from different areas of intensive care ranging from a postanesthesia care unit (PACU) to an ICU for a mixed cohort of patients using the data from a patient data management system (PDMS).

\section{MATERIALS AND METHODS}

The study was approved by the local ethics review board committee of the Charité - University Hospital Berlin and the data safety authorities, which waived the need for individual informed consent (No. EA1/093/12). All adult patients (older than $18 \mathrm{yr}$ ) admitted to an ICU, intermediate care unit (IMCU), or PACU of the Department for Anesthesiology and Operative Intensive Care (Charité, Universitätsmedizin Berlin) between January 1, 2007, and December 31,2008, and discharged alive to a ward in the same hospitalization were included in the analysis.

We retrospectively studied a cohort of patients from four ICUs (named ICU A, B, C, and D), one IMCU, and one PACU. ICU A ( 11 beds) is a medical-surgical ICU, ICU B ( 11 beds) is a surgical ICU for patients after cardiac surgery, ICU C ( 16 beds) is neurological/neurosurgical ICU, and ICU D ( 14 beds) is a medi$\mathrm{cal} /$ surgical ICU specialized on treatment of acute respiratory distress syndrome including extracorporeal lung assist devices.

\section{ICU Organization and Management Policies}

The IMCU (10 beds) predominantly treats postoperative patients and patients from other ICUs, once the patient's condition is stabilized and they comply with the admission criteria for the IMCU. The PACU is eligible for all patients in need of intensive care treatment up to 24 hours or until a bed is available on the ICU, and it is a fully integrated part of our intensive care concept. The staff here is able to perform the same procedures and therapies as in the ICU. The PACU treats patients after surgery but also treats any kind of emergency patients up to 24 hours. This enables patients from the emergency department or patients with a deteriorating cardiopulmonary function on a general ward to be treated in an intensive care setting without any delay.

All units are operated by the Department of Anesthesiology and Operative Intensive Care. A consultant intensivist with a special qualification in intensive care medicine is available 24 hours a day. For every ward, at least one attending physician or in training resident is on available around the clock, working in 8- or 12-hour shifts. There is no reduction in ICU activity or nursing or medical staff at nighttime or during the weekends. ICU physicians, ICU consultants, nursing staff, and the operating surgical team conduct daily rounds. ICU admission and discharge decisions are made by the consultant intensivist in agreement with the surgeon of the patient for postoperative patients. Patients are discharged to a peripheral ward only if there is no organ failure present and the patient's condition no longer demands invasive monitoring. The patients can also be transferred to the IMCU, if the condition demands invasive monitoring or the patients have a single organ failure (except the need for mechanical ventilation).

\section{Data Collection}

Data are collected from vital sign monitors, ventilators, and laboratory data systems and automatically recorded in a PDMS (Copra System GmbH, Sasbachwalden, Germany). The PDMS provides staff with a complete electronic documentation, order entry (medications), documentation of scores, and direct access to laboratory values.

The Simplified Acute Physiology Score (SAPS) II (20) and the Sequential Organ Failure Assessment (SOFA) score (21) are calculated on a daily basis within the PDMS after manual validation of the data by the attending physician. Data recorded at admission and discharge for all patients included gender, age, referring ward, time of admission and discharge, elective or not elective admission, Acute Physiology and Chronic Health Evaluation (APACHE) II score (22). Further variables collected were the vital variables, laboratory values, hours on ventilator for the ICU stay, hours on dialysis, Glasgow Coma Scale (GCS), survival of ICU treatment, and hospital mortality.

The data were extracted from the clinical information system database using MySQL 5.01 (Sun Microsystems GmbH, Kirchheim-Heimstetten, Germany) and then transferred into PASW Statistics 19 (SPSS, Chicago, IL) for further calculations.

Readmission of a patient was defined as a repeated admission to the ICU/IMCU/PACU after discharge of a patient to the general ward who had previously been admitted to one of those units during the same hospitalization period. Transferrals of patients among the ICU, IMCU, and PACU were not considered 
readmissions, and datasets of these patients were aggregated to one clinical case. Planned readmissions after elective surgery were not considered readmissions. Patients, who died within 7 days of discharge on the general ward, were included in the analysis. Only the first readmission from a general ward was included in the analysis. All admission and discharge dates were available from the PDMS. We included PACU patients in the evaluation, because if a patient is in immediate need for intensive care, including patients from general wards, and there is no capacity within the ICU, the patients are transferred to the PACU to stabilize the vital functions until a bed is available in the ICU. Patients transferred to another hospital or different areas of critical care within the same hospital were excluded from the analysis. Also patients who died during the initial ICU stay were not considered in this evaluation, as these patients did not leave the ICU as potential candidates for readmission.

\section{Statistical Analysis}

Data were analyzed using PASW Statistics 19 (SPSS). Continuous data are presented as mean \pm SD and categorical data as number and percentage, unless otherwise indicated. Nonparametric tests of comparison were used for variables evaluated as not being normally distributed. Differences between independent groups were tested using the exact Mann-Whitney $U$ test or Fisher exact test as appropriate. Stepwise, multivariate logistic regression was used to investigate the association between the risk factors for readmission used by Gajic et al and ICU readmission as outcome. Hosmer-Lemeshow goodness-of-fit test was calculated to assess the calibration of the model. Odds ratios (OR) with $95 \% \mathrm{CI}$ and the corresponding $p$-values are given. Receiver operator curve (ROC) analysis was performed to test the discrimination of the SWIFT score with our data.

All statistics were two tailed, and a $p$ value of less than 0.05 was considered statistically significant. All tests should be understood as constituting exploratory data analysis, such that no adjustments for multiple testing have been made.

The SWIFT score was calculated as described in the original publication by Gajic et al (18) for each ICU discharge. Table 1 shows the score calculation worksheet.

As in the original study, the area under the receiver operator curve (AUC) was calculated to describe the discrimination of the model and was compared with the APACHE II scores at the time of ICU discharge.

\section{RESULTS}

The data of 7,175 patients were included in the final calculation of the study (Fig. 1). The readmission-rate was 7.4\%: 6,579 patients were not readmitted to the ICU/IMCU/PACU compared with 528 patients who needed readmission after discharge from the ICU. Table 2 shows the results of the univariate comparison of the groups.

The patients who are readmitted to the ICU in their course of treatment are significantly older and have significantly higher scores that define the severity of disease (APACHE II, SOFA, SAPS) at admission and discharge time of their first ICU stay. The patients with readmission spend more time in the hospital prior to their first ICU admission and have a longer hospital length of stay (LOS).

The source of admission for the initial ICU stay did not show statistical differences $(p=0.055)$ between patients with and without a readmission. Looking at all patients admitted to an ICU, IMCU, or PACU, $35.5 \%$ of the patients were elective admissions to the hospital and intensive care, $46.5 \%$ of the admissions were unplanned or not elective admissions to the hospital, $17.6 \%$ were transferred from another hospital, and $0.4 \%$ of the patients were admitted postpartum.

The last GCS $(p<0.001)$ and the last $\mathrm{PaO}_{2} / \mathrm{FIO}_{2}(p=0.001)$ ratios before discharge from the ICU were higher in the patients who did not need a readmission to the ICU, indicating those patients have better organ function at the time of discharge from the ICU.

The mean SWIFT score for all patients was $14.21( \pm 8.53)$, with a significant difference between the readmitted and nonreadmitted patients (Table 2). During the original development of the SWIFT score, a cut score of 15 was used to discriminate

\section{TABLE 1. Stability and Workload Index for Transfer Score Calculation Worksheet}

\section{Variable SWIFT Points}

Original source of ICU admission

Emergency department

Transfer from a ward or 0 outside hospital

Total ICU length of stay

(duration in days)

$<2$ d

2-10d

$>10 d$

Last measured $\mathrm{PaO}_{2} / \mathrm{FIO}_{2}$ ratio

$\geq 400$

$<400$ and $\geq 150$ 5

$<150$ and $\geq 100$ 10

$<100$

Glasgow Coma Scale at time of ICU discharge

$\geq 14$

11-14

8-11

$<8$

Last arterial blood gas $\mathrm{PacO}_{2}$

$$
\leq 45 \mathrm{~mm} \mathrm{Hg}
$$

$>45 \mathrm{~mm} \mathrm{Hg}$

5

Data taken from Gajic et al (18).

SWIFT $=$ Stability and Workload Index for Transfer. 


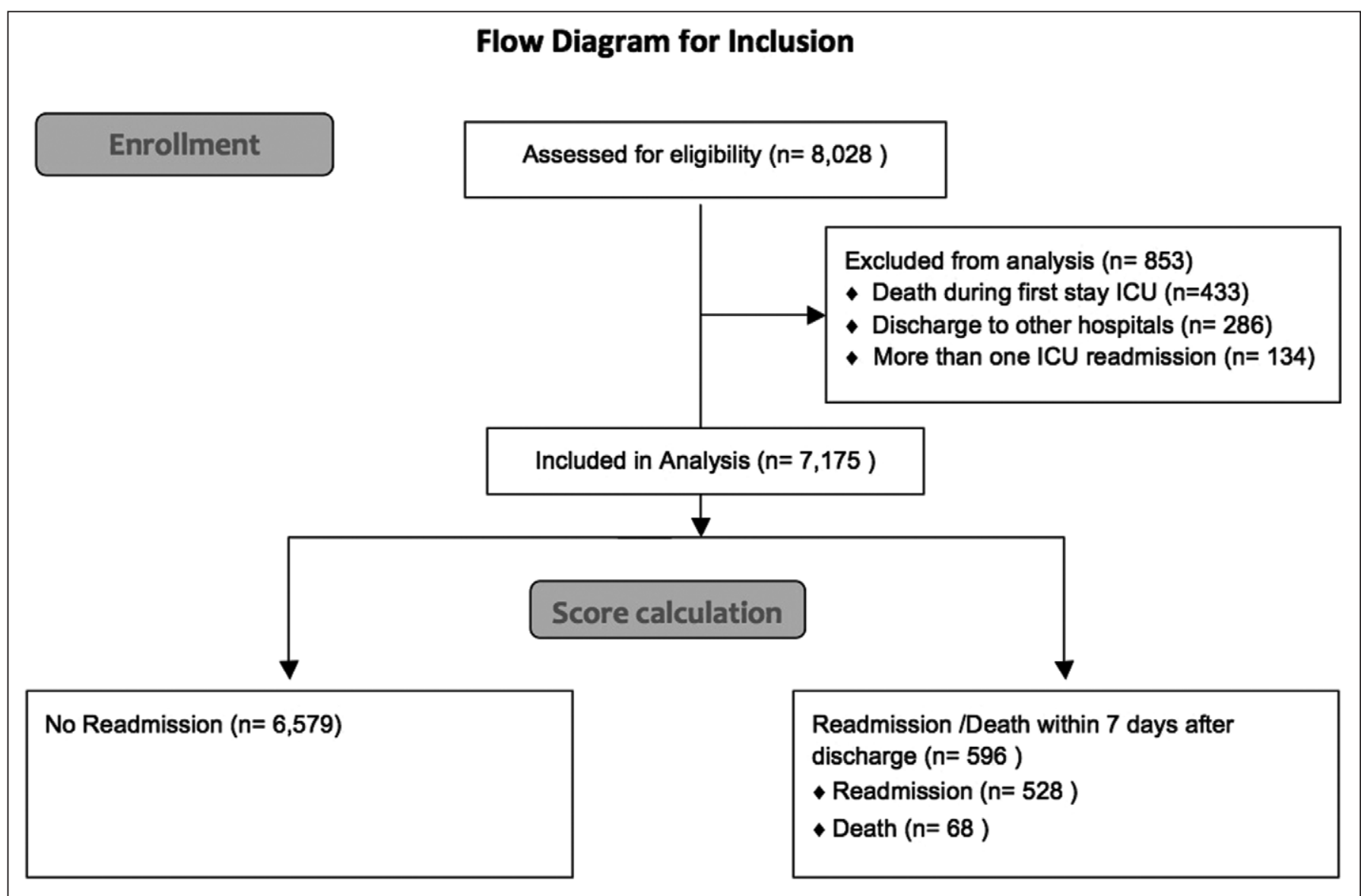

Figure 1. Consort diagram for the inclusion of the patients.

between readmission and nonreadmission. In our population, a cut score of 15 yielded a positive likelihood ratio of 1.36 and a negative likelihood ratio of 0.83 , with specificity of 0.68 and a sensitivity of 0.44 . Using the data of our patients, the determination of the cutoff according to Youden's criterion (maximum [sensitivity + specificity -1 ]) resulted in a cutoff of 13.5 SWIFT points. Table 3 shows the readmission rates according to a cutoff SWIFT score of 15 and our calculated cutoff of 13.5. The cutoff of 13.5 has a sensitivity of 0.552 , a specificity of 0.590 , a positive likelihood ratio of 1.35 , and a negative likelihood ratio of 0.76 .

Figure 2 shows the ROC for the SWIFT score and for the APACHE II score. Overall, the performance of the SWIFT score is poor with an AUC of 0.581 (95\% CI, 0.556-0.605; $p<0.001$ ) using the logistic regression with readmission as outcome and the SWIFT score as the only independent variable. Calibration was poor with a Hosmer-Lemeshow goodness-offit test chi-square of 27.208, $d f 8$, and $p=0.001$ (Cox \& Snell $R^{2}=0.005$, Nagelkerkes $\left.R^{2}=0.011\right)$. SWIFT score was connected with the OR of 1.028 (95\% CI, 1.019-1.037), that is, the probability of readmission would increase by $2.8 \%$ for each point in the SWIFT score. The plot of the observed risk of ICU readmission as the calculated SWIFT score increases is shown in Figure 3.

In the stepwise multivariate logistic regression analysis with the items of the SWIFT score worksheet, the last measured
$\mathrm{PaO}_{2} / \mathrm{FIO}_{2}$ ratio (OR, 1.072; 95\% CI, 1.017-1.129; $p=0.009$ ), the GCS at the time of ICU discharge (OR, 1.019; $95 \% \mathrm{CI}$, $1.004-1.035 ; p=0.016)$, and the last $\mathrm{PaCO}_{2}(\mathrm{OR}, 1.051 ; 95 \%$ CI, 1.001-1.103; $p=0.045$ ) are significantly associated with the risk for readmission to the ICU.

\section{DISCUSSION}

In this retrospective study, we applied a numerical index, the SWIFT score, to a large mixed medical and surgical patient collective from an ICU, IMCU, and a PACU. The SWIFT score was originally developed and validated by Gajic et al (18) and published in 2008. This score was derived on a medical patient group and used in two different validation cohorts of patients. Our results show that certain physiologic variables differ between patients who are readmitted to the ICU and those who are not readmitted. For the SWIFT score, the AUC was 0.581 with a poor calibration in the HosmerLemeshow goodness-of-fit test. In the logistic regression, only the last measured $\mathrm{PaO}_{2} / \mathrm{FIO}_{2}$ ratio, the GCS at the time of ICU discharge, and the last $\mathrm{PaCO}_{2}$ were associated with the risk for readmission to the ICU.

Unfortunately, in the original article by Gajic et al (18), there is no information available, in which way the SWIFT score was determined using the results of the two models of multivariate logistic regression. Furthermore, the use of the same data 


\section{TABLE 2. Univariate Comparison Between Patients With No Readmission and Patients With a Readmission and Death (Without ICU) Within 7 Days After Discharge}

\begin{tabular}{|c|c|c|c|c|c|}
\hline \multirow[b]{4}{*}{ Variable } & \multicolumn{4}{|c|}{ Statistics } & \multirow[b]{4}{*}{$p$} \\
\hline & \multirow{2}{*}{\multicolumn{2}{|c|}{$\begin{array}{l}\text { No Readmission } \\
(n=6,579)\end{array}$}} & & & \\
\hline & & & \multicolumn{2}{|c|}{$\begin{array}{l}\text { All Readmissions } \\
\text { (Including } \\
\text { Death } \leq 7 \mathrm{~d} ; n=596 \text { ) }\end{array}$} & \\
\hline & Mean & SD & Mean & SD & \\
\hline Age (yr) & 63.20 & 16.26 & 66.54 & 15.2 & $<0.001$ \\
\hline $\begin{array}{l}\text { Admission source: emergency } \\
\text { department (\%) }\end{array}$ & $45.4 \%(n=2,988)$ & & $45.1(n=260)$ & & 0.595 \\
\hline Admission APACHE II & 15.88 & 7.7 & 17.70 & 8.0 & $<0.001$ \\
\hline Discharge APACHE II & 12.8 & 8.0 & 14.11 & 8.3 & 0.001 \\
\hline Admission SAPS & 32.38 & 14.9 & 36.8 & 15.8 & $<0.001$ \\
\hline Discharge SAPS & 24.49 & 16.5 & 28.56 & 18.1 & $<0.001$ \\
\hline Admission SOFA & 4.22 & 3.0 & 4.62 & 3.3 & 0.026 \\
\hline Discharge SOFA & 2.86 & 2.9 & 3.31 & 3.1 & 0.002 \\
\hline Duration on ventilator (hr) & 29.1 & 109.0 & 35.12 & 126.3 & 0.041 \\
\hline LOS: ICU days & 2.6 & 7.2 & 3.3 & 7.63 & $<0.001$ \\
\hline LOS: ICU < 2 d (\%) & 78.8 & & 68.6 & & $<0.001$ \\
\hline LOS: ICU 2-10 d (\%) & 15.4 & & 24.5 & & \\
\hline LOS: ICU > 10 d (\%) & 5.8 & & 6.9 & & \\
\hline Last measured $\mathrm{PaO}_{2} / \mathrm{FIO}_{2}$ & 311.7 & 110.8 & 297.3 & 126.2 & 0.001 \\
\hline $\begin{array}{l}\text { Discharge } \mathrm{Paco}_{2} \text { (mm Hg, } \\
\text { maximum value, last day) }\end{array}$ & 41.72 & 8.85 & 42.4 & 8.6 & 0.220 \\
\hline Discharge GCS & 13.75 & 2.6 & 13.22 & 3.2 & $<0.001$ \\
\hline Hospital LOS (d) & 20.95 & 21.7 & 43.11 & 38.38 & $<0.001$ \\
\hline Time in hospital before ICU (d) & 4.25 & 9.38 & 6.72 & 13.9 & $<0.001$ \\
\hline Hospital nonsurvivors & $3.1 \%$ & & $25.7 \%$ & & $<0.001$ \\
\hline Days in hospital after ICU (d) & 16.73 & 18.1 & 36.46 & 33.06 & $<0.001$ \\
\hline SWIFT score & 14.02 & 8.45 & 16.28 & 9.08 & $<0.001$ \\
\hline
\end{tabular}

$\mathrm{APACHE}=$ Acute Physiology and Chronic Health Evaluation; SAPS = Simplified Acute Physiology Score; SOFA = Sequential Organ Failure Assessment; LOS =length of stay; GCS = Glasgow Coma Scale; SWIFT = Stability and Workload Index for Transfer.

Data are from the first stay of the readmitted patients. The $n$ values give the number of available datasets.

for training and testing the performance of the models in the first step leads to an "apparent" accuracy; therefore, the poor results of the two independent datasets are not really astonishing. Many predictor variables with selection procedures judged by significance testing involve multiple comparison problems, which lead to unreliable models. The failure of a model to validate externally could have been avoided by an honest internal validation such as cross-validation or bootstrapping as described by Harrell et al (23) in the development of a prediction model for readmission to the ICU. After all, the use of a Cox regression with a time-dependent covariate "readmission" and further risk factors could result in a better characterization of readmission.

The use of the source of admission might be a possible reason for the poor performance of the model: in the original study, the score had a poor calibration in surgical patients. In the SWIFT model worksheet, 8 points are given for the transfer from a ward or outside hospital to the ICU. Most elective surgical patients are treated on hospital wards prior to surgery, and this might have an effect on the results. Studies have demonstrated a lower risk of readmission for surgical patients (24), some studies have no difference between admission categories (2), 
TABLE 3. Distribution of Patients With Stability and Workload Index for Transfer Cutoff of 15 With No Readmission and With a Readmission or Death Within 7 Days of Discharge

\begin{tabular}{lcc} 
Variable & $\begin{array}{c}\text { No } \\
\text { Readmission, } \\
\boldsymbol{n}(\%)\end{array}$ & $\begin{array}{c}\text { Readmission/ } \\
\text { Death } \leq \mathbf{7} \mathbf{~ d}, \\
\boldsymbol{n}(\%)\end{array}$ \\
\hline SWIFT $<15$ & $4,476(68.8)$ & $336(56.4)$ \\
SWIFT $\geq 15$ & $2,103(32.00)$ & $260(43.6)$ \\
SWIFT $<13.5$ & $3,884(59.0)$ & $267(44.8)$ \\
SWIFT $\geq 13.5$ & $2,695(41.0)$ & $329(55.2)$ \\
Total & $6,579(100.0)$ & $596(100.0)$ \\
\hline
\end{tabular}

$p<0.001$ for both cutoff values.

SWIFT $=$ Stability and Workload Index for Transfer.

and some studies have an increased risk for readmission for surgical patients (4). In our study, $54.6 \%$ of the patients with no readmission are patients with a transfer from a ward or outside hospital compared with $35 \%$ in the Gajic study. For the patients with readmission, the figures are similar for both studies: $54.9 \%$ (our data) versus 57\% (Gajic study).

Our results agree with previous studies, showing that patients with readmissions to the ICU are older and have a higher degree of severity of disease, as measured by the different scores. The definition of reproducible predictors of readmission is limited, as different studies used different designs.

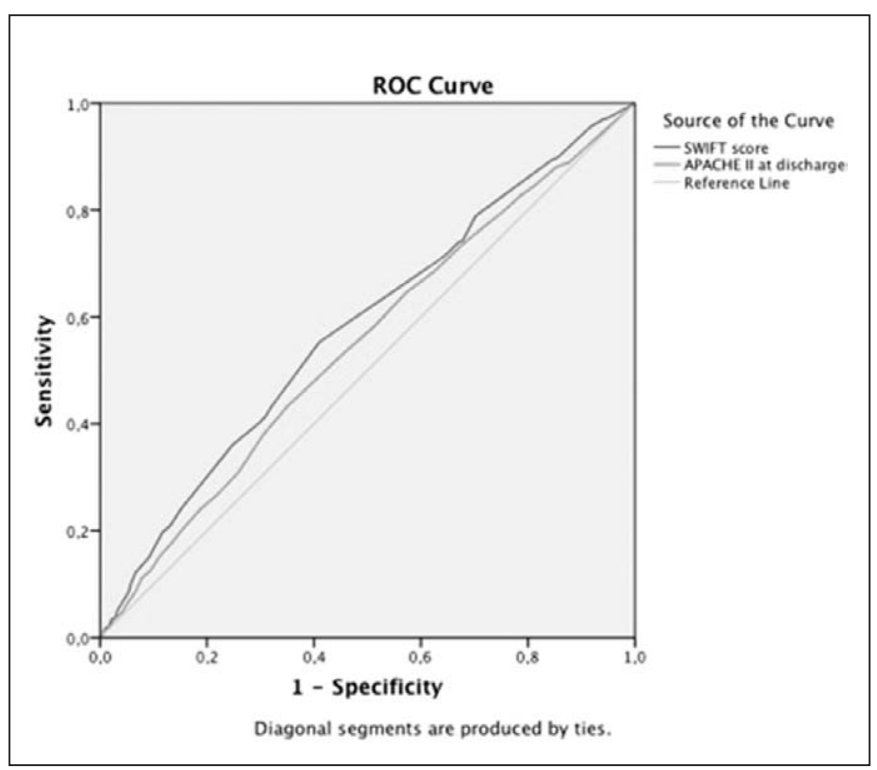

Figure 2. Area under the receiver operator curve $(\mathrm{ROC})$ for Stability and Workload Index for Transfer (SWIFT) score and the Acute Physiology and Chronic Health Evaluation (APACHE) II score, calculated at the time of ICU discharge. Area under the curve: SWIFT score $0.581 ; 95 \% \mathrm{Cl}$, 0.556-0.605; $p<0.001$. APACHE II: 0.548; 95\% Cl, 0.523-0.573; $p$ $<0.001$. Source of the curve: Top line $=$ SWIFT score; middle line $=$ APACHE II at discharge; bottom = reference line.
Only a few of the early studies evaluated patient characteristics, physiology, and treatment status at the time of ICU discharge. Some of the earlier larger epidemiologic studies are limited to the admission day physiological information (1). As Rosenberg et al demonstrated (25), readmissions are determined by the physiological status near the end of the first ICU course, and several other recent studies were able to show that severity of illness as reflected by the extend of physiologic abnormalities at the time of ICU discharge is strongly associated with readmission $(6,9,26)$. However, a meta-analysis of 11 studies came to the conclusion that the risk for readmission increases with severity of illness, independent of the timing of this measurement (admission or discharge) (27). Despite the fact that the results of these studies increase the knowledge about the patients at risk for readmission, evidence-based discharge criteria for patient groups at risk, like sepsis or mechanically ventilated patients, which could prevent adverse events after ICU discharge, deserve further studies.

A valid score might help differentiate between patients who can be discharged safely to a general ward, to a higher acuity step down unit, who need increased supervision in the general care area with overlapping rounds by an ICU team, or who are in need for further ICU treatment. A requirement for the development of a valid discharge score is the definition of general discharge criteria from the ICUs. These criteria will also depend on the local conditions. In the study by Kramer (6) on ICU readmissions, for example, 5.2\% of the patients had a GCS of 6 or lower on the day of ICU discharge and $6.7 \%$ of the patients were sedated, and it was unable to assess the GCS on the discharge day. In many ICUs, these patients would

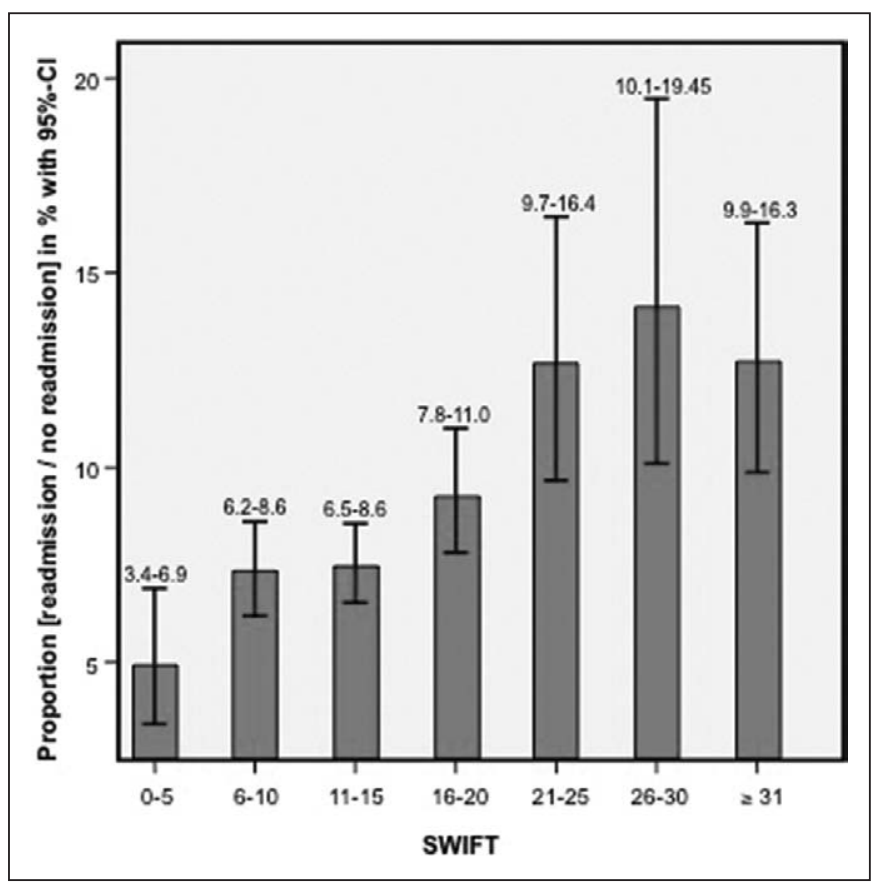

Figure 3. Plot of the observed risk of ICU readmission as the calculated Stability and Workload Index for Transfer (SWIFT) score increases by increments of 5 (with $95 \% \mathrm{Cls}$ ). 
not have fulfilled the discharge criteria. It should be considered that scores like the SWIFT score that was developed and independently validated in the United States and the Netherlands cannot be applied to all other countries. International differences in healthcare systems often lead to conflicting results in validation of other scoring systems.

Ourstudy has severallimitations, which need to be considered in the interpretation of the results. It is a retrospective analysis of prospectively collected data and therefore cannot demonstrate causality. One limitation is that not all variables are available for all patients, for example, certain laboratory values like the $\mathrm{PaCO}_{2}$, which is only measured if the patient has an arterial line and has an indication for these measurements. During the calculation of the statistics, the patients with missing values can be treated in two ways: the patients are not included in the calculations or as done by Gajic et al, the values are assumed to be normal. Both ways of handling missing data might influence the results. We did not differentiate between the different types of ICU, surgical, medical, or neurological, as most of the ICUs are able to treat all kinds of patients. Unlike other studies, we included all patients in need of intensive care, independently of ICU LOS. This makes some comparisons difficult, as other studies excluded patients who were in the ICU for less than 4 hours (6) or included all patients independent of ICU LOS (7). No clinical data, for example, scores are collected in a database on general wards. Thus, it is not possible to determine factors arising after discharge of patients. Like in other studies using large databases, patients discharged with treatment limitations are not identified and are part of the cohort. They have less likelihood of being readmitted but might influence the number of hospital deaths. Expectation of outcome at the time of discharge is also not known. Another limitation of our study is that there was no specific evaluation of case mix or other disease-related information. In clinical context, this information is difficult to obtain, as besides the main medical focus all the evaluated ICUs treat patients in need of intensive care irrespective of the underlying disease.

\section{CONCLUSION}

The results from this study show that there are patients with an increased risk for readmission. Further studies, as well as cost/ benefit analysis, are needed to investigate whether discharge decisions based on certain scores, which include defined physiological variables, will influence the number of unplanned ICU readmission or improve outcome. The impact of clinical outreach teams on readmissions has to be further investigated, and a definition of what variables need to be monitored for defined patient groups is needed. Nevertheless, readmission rates and unexpected death after ICU treatment may be a relevant quality marker, particularly on a local level.

\section{ACKNOWLEDGMENTS}

We thank Gerda Siebert and Dr. Brigitte Wegner for their statistical advice and help and Stefan Rattey for help in the data acquisition.

\section{REFERENCES}

1. Cooper GS, Sirio CA, Rotondi AJ, et al: Are readmissions to the intensive care unit a useful measure of hospital performance? Med Care 1999; 37:399-408

2. Metnitz PG, Fieux F, Jordan B, et al: Critically ill patients readmitted to intensive care units-lessons to learn? Intensive Care Med 2003; 29:241-248

3. Alban RF, Nisim AA, Ho J, et al: Readmission to surgical intensive care increases severity-adjusted patient mortality. J Trauma 2006; 60:1027-1031

4. Ho KM, Dobb GJ, Lee KY, et al: The effect of comorbidities on risk of intensive care readmission during the same hospitalization: $A$ linked data cohort study. J Crit Care 2009; 24:101-107

5. Renton J, Pilcher DV, Santamaria JD, et al: Factors associated with increased risk of readmission to intensive care in Australia. Intensive Care Med 2011; 37:1800-1808

6. Kramer AA, Higgins TL, Zimmerman JE. Intensive care unit readmissions in U.S. hospitals: Patient characteristics, risk factors, and outcomes. Crit Care Med 2012; 40:3-10

7. Brown SE, Ratcliffe SJ, Kahn JM, et al: The epidemiology of intensive care unit readmissions in the United States. Am J Respir Crit Care Med 2012; 185:955-964

8. Chrusch CA, Olafson KP, McMillan PM, et al: High occupancy increases the risk of early death or readmission after transfer from intensive care. Crit Care Med 2009; 37:2753-2758

9. Durbin CG Jr, Kopel RF: A case-control study of patients readmitted to the intensive care unit. Crit Care Med 1993; 21:1547-1553

10. Utzolino S, Kaffarnik M, Keck T, et al: Unplanned discharges from a surgical intensive care unit: Readmissions and mortality. J Crit Care 2010; 25:375-381

11. Chen LM, Martin CM, Keenan SP, et al: Patients readmitted to the intensive care unit during the same hospitalization: Clinical features and outcomes. Crit Care Med 1998; 26:1834-1841

12. Miles TA, Lowe J: Are unplanned readmissions to hospital really preventable? J Qual Clin Pract 1999; 19:211-214

13. Ashton CM, Del Junco DJ, Souchek J, et al: The association between the quality of inpatient care and early readmission: A meta-analysis of the evidence. Med Care 1997; 35:1044-1059

14. Franklin C, Jackson D: Discharge decision-making in a medical ICU: Characteristics of unexpected readmissions. Crit Care Med 1983; $11: 61-66$

15. Fernandez R, Serrano JM, Umaran I, et al; Sabadell Score Study Group: Ward mortality after ICU discharge: A multicenter validation of the Sabadell score. Intensive Care Med 2010; 36: 1196-1201

16. Ball C, Kirkby M, Williams S: Effect of the critical care outreach team on patient survival to discharge from hospital and readmission to critical care: Non-randomised population based study. BMJ 2003; 327:1014

17. Williams TA, Leslie G, Finn J, et al: Clinical effectiveness of a critical care nursing outreach service in facilitating discharge from the intensive care unit. Am J Crit Care 2010; 19:e63-e72

18. Gajic O, Malinchoc M, Comfere TB, et al: The Stability and Workload Index for Transfer score predicts unplanned intensive care unit patient readmission: Initial development and validation. Crit Care Med 2008; 36:676-682

19. Chandra S, Agarwal D, Hanson A, et al: The use of an electronic medical record based automatic calculation tool to quantify risk of unplanned readmission to the intensive care unit: $A$ validation study. J Crit Care 2011; 26:634.e9-634.e15

20. Le Gall JR, Lemeshow S, Saulnier F: A new Simplified Acute Physiology Score (SAPS II) based on a European/North American multicenter study. JAMA 1993; 270:2957-2963

21. Vincent JL, Moreno R, Takala J, et al: The SOFA (Sepsis-related Organ Failure Assessment) score to describe organ dysfunction/failure. On behalf of the Working Group on Sepsis-Related Problems of the European Society of Intensive Care Medicine. Intensive Care Med 1996; 22:707-710 
22. Knaus WA, Draper EA, Wagner DP, et al: APACHE II: A severity of disease classification system. Crit Care Med 1985; 13: 818-829

23. Harrell FE Jr, Lee KL, Mark DB: Multivariable prognostic models: Issues in developing models, evaluating assumptions and adequacy, and measuring and reducing errors. Stat Med 1996; 15:361-387

24. Frost SA, Tam V, Alexandrou E, et al: Readmission to intensive care: Development of a nomogram for individualising risk. Crit Care Resusc 2010; 12:83-89
25. Rosenberg AL, Hofer TP, Hayward RA, et al: Who bounces back? Physiologic and other predictors of intensive care unit readmission. Crit Care Med 2001; 29:511-518

26. Snow N, Bergin KT, Horrigan TP: Readmission of patients to the surgical intensive care unit: Patient profiles and possibilities for prevention. Crit Care Med 1985; 13:961-964

27. Frost SA, Alexandrou E, Bogdanovski T, et al: Severity of illness and risk of readmission to intensive care: A meta-analysis. Resuscitation $2009 ; 80: 505-510$ 